\title{
Geodesic Active Contours with Adaptive Neighboring Influence
}

\author{
Huafeng Liu ${ }^{1,2}$, Yunmei Chen ${ }^{3}$, Hon Pong $\mathrm{Ho}^{2}$, and Pengcheng $\mathrm{Shi}^{2}$ \\ 1 State Key Laboratory of Modern Optical Instrumentation, \\ Zhejiang University, Hangzhou, China \\ 2 Department of Electrical and Electronic Engineering, \\ Hong Kong University of Science and Technology, Hong Kong \\ 3 Department of Mathematics, University of Florida, Gainesville, USA
}

\begin{abstract}
While geometric deformable models have brought tremendous impacts on shape representation and analysis in medical image analysis, some of the remaining problems include the handling of boundary leakage and the lack of global understanding of boundaries. We present a modification to the geodesic active contour framework such that influence from local neighbors of a front point is explicitly incorporated, and it is thus capable of robustly dealing with the boundary leakage problem. The fundamental power of this strategy rests with the local integration of evolution forces for each front point within its local influence domain, which is adaptively determined by the local level set geometry and image/prior information. Due to the combined effects of internal and external constraints on a point and the interactions with those of its neighbors, our method allows stable boundary detection when the edge information is noisy and possibly discontinuous (e.g. gaps in the boundaries) while maintaining the abilities to handle topological changes, thanks to the level set implementation. The algorithm has been implemented using the meshfree particle domain representation, and experimental results on synthetic and real images demonstrate its superior performance.
\end{abstract}

\section{Introduction}

Shape recovery has been one of the most active research areas in medical image analysis because of its practical importance and theoretical challenges. Over the last two decades, various parametric and geometric deformable models have gained much popularity [47. Geodesic active contours have been proposed [1] to connect classical parametric snakes based on energy minimization to geometric active contours based on the theory of curve evolution [8], and thus maintain the desirable properties of allowing topological changes during the curve evolution process. Nevertheless, these geometric snakes still have certain drawbacks, i.e. they face difficulties in handling weak edges/gap problems 110 where the evolving contour cannot stick to the object boundary and would simply leak through the gaps, they are very sensitive to local minima in noisy images [1].

In order to overcome these problems, considerable progress has already been made through the use of additional force (or energy) terms. An extra stopping 

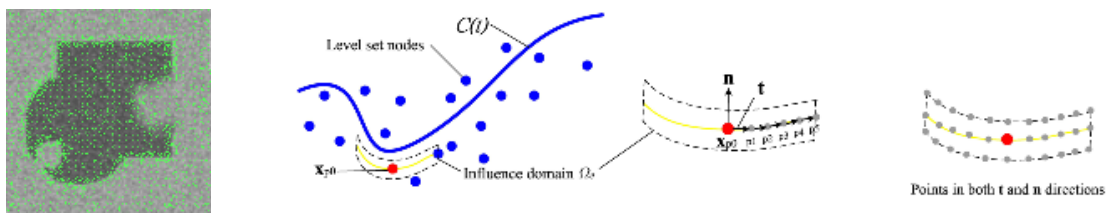

Fig. 1. 1st: adaptive point-based domain representation; 2nd: influence domain (and the associated other points) of the red point; 3rd: closeup which illustrates the generation of neighboring points in the tangent direction (we generate the points $p_{n}$ through $\mathbf{x}_{p_{n}}=\mathbf{x}_{p_{(n-1)}}+\frac{t_{s c a l e}}{|\kappa|} \mathbf{t}$, with $\kappa$ the curvature and $\mathbf{t}$ the tangent vector of point $\left.p_{(n-1)}\right)$; 4th: generated neighboring points in both normal and tangent directions (and thus the influence domain) for the red point.

term for pulling back the contour if it passes the boundary was investigated in [1], although this formulation is still susceptible to boundary leakage problem. A weighted area functional force has been introduced to help the snake be more robust with respect to small gap problem [10. Diffused external data forces, such as the gradient vector flow (GVF), have also been adopted to deal with the leakage problem and noisy data 12 . And boundary and region information has been integrated under a curve-based minimization framework 9]. These later approaches own the benefits provided by external force potential field in achieving a larger capture range and robustness against the boundary leakage problem 912. More recently, a region-aided geometric snake, which integrates gradient flow forces with region vector flow forces obtained through the diffusion of the region segmentation map, has been developed and implemented within level set platform [11. These integrated forces give another way to be more robust toward weak edges. Similar region-based strategies have been explored by other works as well [2].

We realize that the boundary leakage problem can be more readily solved if the behavior of individual curve point is constrained by local edge information of itself and that of its neighboring points. These inter-point relationships act as diffused local internal energy, which would make the snakes less sensitive to noisy or broken edges. Thus, our aim is to develop a robust segmentation framework that imposes adaptive local inter-point constraints into geodesic active contours. Instead of using additional external forces, we make use of the image data at the adaptively determined local support domain around each point of interest, which effectively enlarges the capture range of each point to have a better local understanding of the image information within its local neighborhood. In other words, we modify the image forces on each point of the geodesic contour in a way such that it is capable of providing sufficient information to define a desired segmentation which is robust against boundary leakage and noise impact. One key issue is the proper determination of the local neighborhood of each curve point such that the integration of information can be performed. In this paper, we present a numerical implementation of the strategy on the meshfree particle representation of the evolution domain, where the local neighbor is adaptively selected based on image data and curve geometry. 


\section{Methodology}

\subsection{Geodesic Active Contours (GACs)}

Let us consider an active contour $C(s)$ parameterized by $s \in[0,1]$. It has been known that the problem of boundary detection can be casted into the problem of minimizing the curve length in Riemannian space $\min \int_{0}^{1} g(|\nabla I(C(s))|)\left|C^{\prime}(s)\right| d s$, where $g($.$) is a strictly decreasing edge-function such that g(0)=1$ and $\lim _{x->\infty}$ $g(x)=0$ [1]. Now we represent the evolving contour $C$ implicitly as the zero level set of a scalar Lipschitz function $\phi: C(t)=\{x \mid \phi(x, t)=0\}$. The corresponding energy over the image domain $\Omega$ in terms of level set function $\phi$ becomes:

$$
E(\phi)=\int_{\Omega} g(|\nabla I(x)|)|\nabla H(\phi(x))| d x
$$

where $H(x)$ is the Heaviside function, that is $H(x)=1$ if $x>=0$, and $H(x)=0$ if $x<0$, and let $\delta_{\varepsilon}(x)=H^{\prime}(x)$ be the Dirac measure. Then, the length of zero level set is given by $\int_{\Omega}|\nabla H(\phi(x))|=\int_{\Omega} \delta_{\varepsilon}(\phi)|\nabla \phi(x)|$. The energy can be rewritten:

$$
E(\phi)=\int_{\Omega} \delta_{\varepsilon}(\phi) g(|\nabla I(x)|)|\nabla \phi(x)| d x
$$

The minimization process can be achieved by solving the Euler-Lagrange equation, obtained by minimizing Eqn. (2) with respect to $\phi$ and parameterizing the descent directions by an artificial time $t$ :

$$
\frac{\partial \phi}{\partial t}=\delta_{\varepsilon}(\phi) \operatorname{div}\left(g(|\nabla I(x)|) \frac{\nabla \phi}{|\nabla \phi|}\right)
$$

We reach the following equations by expanding the divergence term and replacing $\delta_{\varepsilon}(\phi)$ by $\nabla \phi[13$ :

$$
\frac{\partial \phi}{\partial t}=g(|\nabla I(x)|)|\nabla \phi| \operatorname{div}\left(\frac{\nabla \phi}{|\nabla \phi|}\right)+\nabla g(|\nabla I(x)|) \cdot \nabla \phi
$$

where $\frac{\nabla \phi}{|\nabla \phi|}$ denotes the unit normal vector. The divergence of the unit normal vector div $\left(\frac{\nabla \phi}{|\nabla \phi|}\right)$ represents the curvature of the current point.

\subsection{GACs with Adaptive Neighboring Influence (GAC-ANI)}

The main idea in the GAC-ANI formulation is that centered at each front point, there is an influence domain $\Omega_{e}$ which contains points that have effects on the evolution of the concerned front point. Hence, each front point moves under the influence of two forces: the typical data force provided by image information such as GVF, and the neighborhood force due to the interaction of the point with other points in the influence domain. With proper formulation of the 
neighboring interactions, front points at the weak edges or gaps will be dominated by the neighborhood force such that the front would be discouraged from leaking through the boundary. For front points with good data force, their movement is still mostly controlled by image information and thus would stick to the object boundary exhibited in the image. Due to the combined effects of data constraints and interactions with the neighboring points, GAC-ANI exhibits robustness against boundary leakage while maintaining the desired geometrical characteristics of GACs.

While there could be many ways to incorporate the inter-point relationship into the GAC-ANI formulation, one simple way of enforcing neighborhood influence is to replace the edge function $g(x)$ in Equation (2) by $G(x)=\int_{\Omega_{e}} \mathbf{N} g(y) d y$, where $\mathbf{N}$ is a shape function that assigns proper weights to each point within the influence domain. In this sense, the evolution force on point $x$ is now constrained by the image data at the point itself and at the other points within its influence domain. The modified objective function of GAC-ANI now becomes:

$$
E(\phi)=\int_{\Omega} \delta_{\varepsilon}(\phi)\left(\int_{\Omega_{e}} \mathbf{N} g(y) d y\right)|\nabla \phi(x)| d x=\int_{\Omega} \delta_{\varepsilon}(\phi) G(x)|\nabla \phi(x)| d x
$$

The evolution equation related to the Euler-Lagrange equation for Eqn. (5) is:

$$
\left.\frac{d}{d \tau} E(\phi+\tau \Phi)\right|_{\tau=0}=0
$$

Now, let us take care of the left hand side of the equation:

$$
\frac{d}{d \tau} \int_{\Omega} \delta_{\varepsilon}(\phi+\tau \Phi) G(x)|\nabla \phi+\tau \nabla \Phi| d x=-\int_{\Omega} \delta_{\varepsilon}(\phi) \operatorname{div}\left(G(x) \frac{\nabla \phi}{|\nabla \phi|}\right) \Phi d x(7)
$$

Finally, the following Euler-Lagrange equation can be achieved:

$$
\delta_{\varepsilon}(\phi) \operatorname{div}\left(G(x) \frac{\nabla \phi}{|\nabla \phi|}\right)=0
$$

It is common to expand the divergence term to obtain the alternative equation:

$$
\delta_{\varepsilon}(\phi) G(x) \operatorname{div}\left(\frac{\nabla \phi}{|\nabla \phi|}\right)+\delta_{\varepsilon}(\phi) \nabla G(x) \cdot \frac{\nabla \phi}{|\nabla \phi|}=0
$$

where $\nabla G=\int_{\Omega_{e}} \nabla \mathbf{N} g(y) d y$. The steady state solution of the above equation results in the GAC-ANI formulation in level set representation, where a standard rescaling can be made through replacing $\delta_{\varepsilon}(\phi)$ by $|\nabla \phi|[13$ :

$$
\frac{\partial \phi}{\partial t}=G(x)|\nabla \phi| \operatorname{div}\left(\frac{\nabla \phi}{|\nabla \phi|}\right)+\nabla G(x) \cdot \nabla \phi
$$


Discussions. It should be noted that the most attractive property of the above GAC-ANI formulation is that the influence domain $\Omega_{e}$ controls the trade-off between a parametric snake and a geodesic snake at the front point.

Consider the case where the size of the local influence domain is approaching zero, e.g. the level set value of the point is mainly determined by itself. Hence, Eqn. (10) goes back to the standard geodesic active contours. On the other hand, if we enlarge the influence domain to cover the entire curve, the integration is then taken over the whole contour. Perceptually, the behavior of all curve points are now inter-related, and we effectively have a parametric deformable model instead. In practice, influence domains of different sizes generate different $G$ and $\nabla G$, which in turn are suitable for different situations. For example, large influence domains are effective in robust segmentation of noisy images or object with broken edges. On the other hand, small influence domains are needed for object boundaries with many fine details. Schemes to adaptively determine the sizes of the influence domains will be discussed in the following section.

\subsection{Numerical Implementations of GAC-ANI}

We have implemented the GAC-ANI on the evolution domain represented by adaptively distributed meshfree particles [3. Here, we want to point out that any numerical implementations such as traditional finite difference schemes can also be used for GAC-ANI without any fundamental algorithmic modifications.

Let $\phi(\mathbf{x}, t=0)= \pm d$, where $\pm d$ is the signed distance to the interface. The level set updating procedures on the meshfree particle domain are:

1. Initialization: Initialize $\phi(\cdot, 0)$ to be the signed distance function.

2. Domain Representation: We adopt an adaptive point distribution scheme to represent the domain by meshfree particles (see Fig. 1 for an example). The point distribution is adaptive towards both local level set geometry and image gradient, and it allows extremely convenient enhancement/reduction of curve precision by simply putting more/fewer points on the computation domain. A detailed discussion of this scheme can be found in 3 . Then, find all the points in the narrow band of the current zero level set.

3. Influence Domain Generation: Generate a proper influence domain for each point within the narrow band (see detailed discussion later).

4. Shape Function Construction: Here, we use the concept of moving least squares (MLS) to construct the shape functions. It is assumed the current active point $\mathbf{x}$ with its neighboring nodes $\mathbf{x}_{I}, I=1,2, \ldots, n$ are given in the influence domain. Following the work in [5], the MLS-derived shape function is $N_{I}(\mathbf{x})=\sum_{j}^{m} p_{j}(\mathbf{x})\left(\mathbf{A}^{-1}(\mathbf{x}) \mathbf{B}(\mathbf{x})\right)_{j I}=\mathbf{p}^{T} \mathbf{A}^{-1} \mathbf{B}_{I}$, with $\mathbf{A}(\mathbf{x})=$ $\sum_{I} w\left(\mathbf{x}-\mathbf{x}_{I}\right) \mathbf{p}\left(\mathbf{x}_{I}\right) \mathbf{p}^{T}\left(\mathbf{x}_{I}\right), \mathbf{B}_{I}=w\left(\mathbf{x}-\mathbf{x}_{I}\right) \mathbf{p}\left(\mathbf{x}_{I}\right), \mathbf{B}(\mathbf{x})=\left[\mathbf{B}_{1}, \mathbf{B}_{2}, \ldots, \mathbf{B}_{n}\right]$. $\mathbf{p}(\mathbf{x})$ is polynomial basis functions, and $w\left(\mathbf{x}-\mathbf{x}_{I}\right)$ is the weighting function.

5. Evaluation of Integrals: To update level set function of Equation (10), matrices $G$ and $\nabla G$ need to be calculated. That is, one needs to integrate over the influence domain. This can be carried out through numerical techniques which approximate a continuous integral over $\Omega_{e}$ into a discrete sum: 
$\int_{\Omega_{e}} f(\xi)=\sum_{l=1}^{n_{q}} w_{l} f\left(\xi_{l}\right)$, where $n_{q}$ is number of quadrature points, $\xi_{l}$ is the coordinates of sampling point $l$, and $w_{l}$ is the corresponding weighting factor. Here, we use the Gaussian quadrature technique which is the most commonly used integration scheme in meshfree particle methods [6].

6. Updating Procedure: Update level set function $\phi$ using Equation (10).

7. Reinitialization: Re-initialize $\phi(\cdot, t+1)$ to be the signed distance function of its zero level set.

8. Convergence Test: Set proper convergence criterion to test whether the zero level set reaches object boundary. If no, go back to step 2.

Influence Domain Determination. In the level set formulation based on distance measure, there is an entire family of isocontours of different level set values (although only one of which is the zero level set). For each data point in the narrow band, or a node, its all important influence domain $\Omega_{e}$ is determined by a data-driven local operation. And the geometry of the resulting influence domain adapts to the isocontour segment to which it belongs (see Fig. 1 for an illustration).

First, we calculate the image gradient magnitude $\left|\nabla I\left(\mathbf{x}_{n b}\right)\right|$ within the narrowband and normalize them to $[0,1]$, where $\mathbf{x}_{n b}$ is the narrow band node points set. Starting with an arbitrary narrow band point $\mathbf{x}_{n b(i)}$ (the red point in Fig. 1), let $\mathbf{x}_{i}=\mathbf{x}_{n b(i)}$ and tag it as active. We then compute the normal vector $\mathbf{n}$, the tangent vector $\mathbf{t}$, and curvature $\kappa$ of the active point. Adding a tangent virtual node along the tangent direction use $\mathbf{x}_{i}=\mathbf{x}_{i}+\frac{t_{\text {scale }}}{|\kappa|} \mathbf{t}$ (adding the opposite point by $\left.\mathbf{x}_{i}=\mathbf{x}_{i}-\frac{t \text { scale }}{|\kappa|} \mathbf{t}\right)$, where $t_{\text {scale }}$ is the tangential scale factor. This way, the higher the curvature, the closer the added point will be to the active point, which will in turn guarantee that fine shape details will be preserved. We then tag the new added point as active point. This process is executed iteratively until the number of added points has reached a specified limit, which is determined

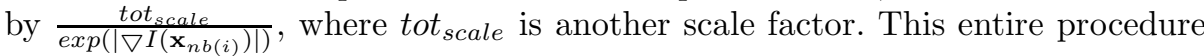
implies that for low image gradient node, many virtual points will be added in the tangent direction (large $\Omega_{e}$ dimension size in the tangent direction). Of course for high image gradient node, there will be few virtual points added and the $\Omega_{e}$ dimension size in the tangent direction will be small. In the same fashion, virtual nodes in normal direction can be added using a similar scheme by $\mathbf{x}_{i} \pm n_{\text {scale }}\left|\nabla I\left(\mathbf{x}_{i}\right)\right| \mathbf{n}$ where $n_{\text {scale }}$ is the normal direction scale factor.

\section{Experiments and Results}

In Fig. 2, comparison is made between traditional GAC and GAC-ANI on their ability to deal with boundary gaps. The test object contains a big blurred area on the right boundary and a small blurred area on the lower left boundary. Clearly, the traditional GAC curve keeps shrinking and leaks through the broken edges, while GAC-ANI does not suffer from such leakage problem and converges to the true boundary since the neighborhood point information offers useful 

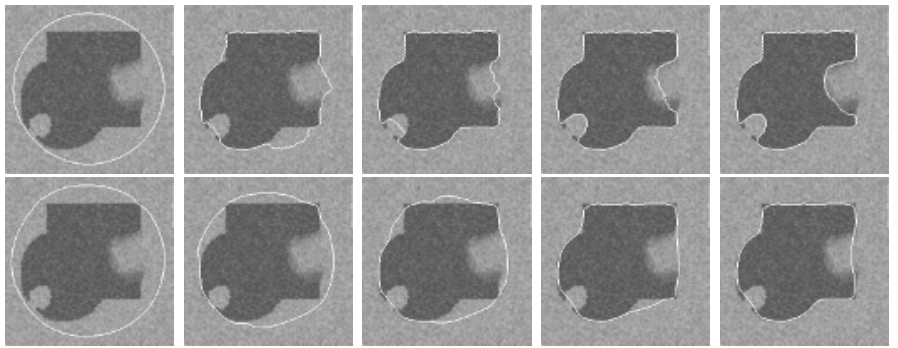

Fig. 2. Segmentation of noisy synthetic image with boundary occlusion: traditional level set implemented on finite difference grid (top); GAC-ANI on adaptive point cloud.
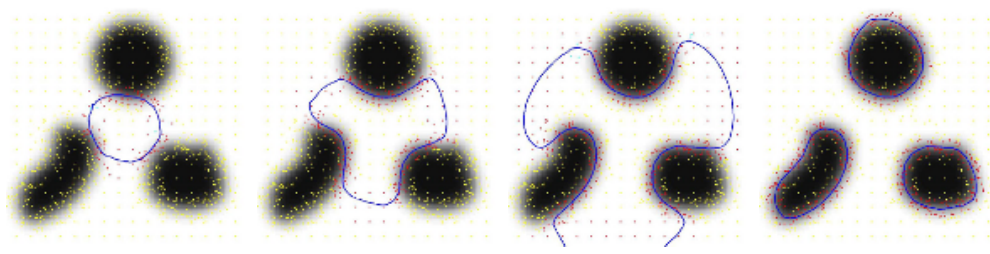

Fig. 3. The ability of the GAC-ANI to handle topological changes
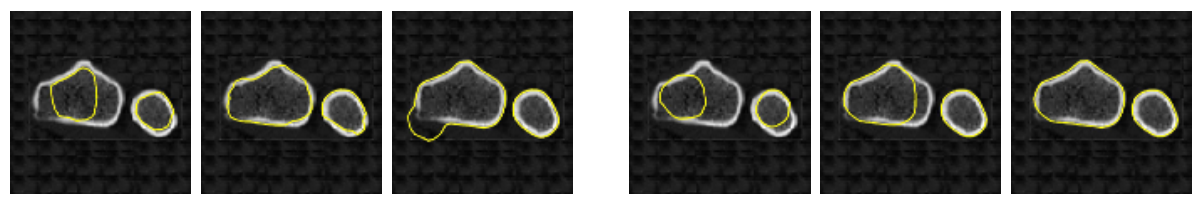

Fig. 4. Segmentation process on the bone CT image: GAC (left) and GAC-ANI (right)
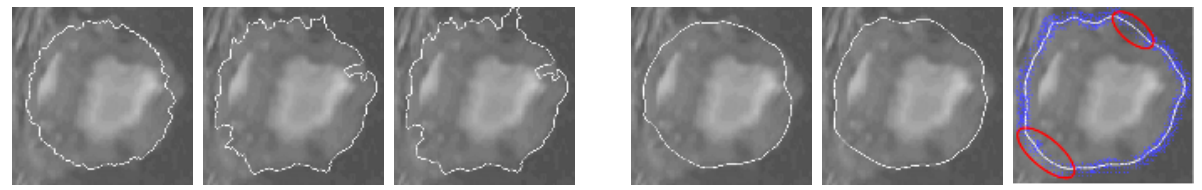

Fig. 5. Epicardial segmentation process on canine MRI image: traditional GAC (left three) and GAC-ANI (right three). The red circles highlight the ill-defined boundary areas (upper-right: image void caused by implanted marker; lower-left: weak contrast between myocardium and background tussue) where neighboring influence is dominant.

expanded view on the image boundaries. Each contour point of the GAC-ANI belonging to the blurred area is adaptively assigned large influence domain and thus detects the boundary properly, while front point elsewhere is determined to have very small influence domain and thus behaves just like the traditional level set point. In Fig. 3. starting from a single front, GAC-ANI manages to split and capture all the boundaries of three objects, just like a traditional GAC. During 
the segmentation process, as the front moves, the nodes in the narrow band are adaptively constructed depending on local image (red points) and geometry (cyan points). Finally, we show the segmentation results on several real medical images. In the bone CT image segmentation (Fig. 4), the low-left corner of the big bone has a relatively weak edge. While the traditional GAC leaks through the edge (left figures), GAC-ANI properly stops at that part of the edge (right figures). In the difficult task of epicardial segmentation from the canine cardiac MRI image (Fig. [5), the ill-defined epicardium from its background and the void caused by implanted imaging-opaque markers make the traditional GAC fail to produce appropriate definition of the boundary, while the proposed GAC-ANI yields proper, smooth segmentation result.

Acknowledgement. This work is supported in part by the 973 Program of China (2003CB716104), by the HKRGC-CERG HKUST6252/04E, by the NSF of China(60403040).

\section{References}

1. V. Caselles, R. Kimmel, and G. Sapiro. Geodesic active contours. International Journal of Computer Vision, 22(1):61-79, 1997.

2. T. Chan and L. Vese. Active contours without edges. IEEE Transactions on Image Processing, 10(2):266-277, 2001.

3. H. Ho, Y. Chen, H. Liu, and P. Shi. Level set active contours on unstructured point cloud. In IEEE Computer Vision and Pattern Recognition, 2005.

4. M. Kass, A. Witkin, and D. Terzopoulos. SNAKES: Active contour models. International Journal of Computer Vision, 1:321-332, January 1988.

5. P. Lancaster and K. Salkauskas. Surface generated by moving least squares methods. Mathematics of Computation, 37(155):141-158, 1981.

6. H. Liu and P. Shi. Meshfree representation and computation: Applications to cardiac motion analysis. In Information Processing in Medical Imaging, pages 560-572, Ambleside, U.K, July 2003.

7. R. Malladi, J.A. Sethian, and B.C. Vemuri. Shape modeling with front propagation: A level set approach. IEEE Transactions on Pattern Analysis and Machine Intelligence, 17(2):158-175, 1995.

8. S. Osher and J.A. Sethian. Fronts propagating with curvature-dependent speed: Algorithms based on hamilton-jacobi formulations. Journal of Computational Physics, 79:12-49, 1988.

9. N. Paragios and R. Deriche. Coupled geodesic active regions for image segmentation: a level set apporach. In Proceedings of the Europe Conference on Computer Vision, pages 224-240, 2000.

10. K. Siddiqi, Y. Lauziere, A. Tannenbaum, and S. Zucker. Area and lengthminimizing flows for shape segmentation. IEEE Transactions on Image Processing, 7:433-443, 1998.

11. X. Xie and M. Mirmehdi. RAGS: Region-aided geometric snake. IEEE Transactions on Image Processing, 13(5):640-652, May 2004.

12. C. Xu and J. Prince. Generalized gradient vector flow external forces for active contours. Signal Processing, 71(2):131-139, 1998.

13. H. Zhao, T. Chan, B. Merriman, and S. Osher. A variational level set approach to multiphase motion. Journal of Computational Physics, 127:179-185, 1996. 